\title{
Errichtung von zusätzlichen Entspannungsbrunnen beim Erddamm Durlaßboden
}

\begin{abstract}
Zusammenfassung: Bei dem auf Überlagerungsmaterial gegründeten Staudamm Durlaßboden zeigt sich durch die begrenzte Abdichtung eine markante Beeinflussung der im luftseitigen Vorland auftretenden Druckhöhen und Sickerwasseraustritte in Abhängigkeit von der Stauhöhe im Speicher.

Eine projektsgemäße Entlastung am luftseitigen Dammfuß erfolgte seit Errichtung des Dammes - die im Bereich des Unterwassergrabens auftretenden hohen Porenwasserdrücke konnten hingegen erst durch die zusätzlich errichteten Entspannungsbrunnen entsprechend abgesenkt werden. Durch diese Maßnahme konnten die Grundbruchsicherheiten und Austrittsgradienten in diesen Abschnitt entscheidend verbessert werden und erhöhen damit die Sicherheit der Gesamtanlage deutlich.
\end{abstract}

Additional relief wells for the

Durlassboden earth dam

Summary: As the Durlassboden earth dam is founded on overburden material, the impervious elements are of limited depth. This has led to variations in downstream pressure heads and seepage flows as a function of reservoir water levels.

The originally intended relief was provided at the downstream dam toe after the construction of the dam, but additional relief wells were needed to reduce the high pore water pressures occurring in the tailwater trench. These measures have substantially improved the seepage gradients and the safety against base failure in this area and thus significantly increase the safety of the entire facility.

\section{Einleitung}

Der $85 \mathrm{~m}$ hohe Staudamm Durlaßboden wurde in den Jahren 1964-1966 nahe der Ortschaft Gerlos als Erdschüttdamm mit einem zentralen Dichtungskern aus Lehm errichtet (Abb. 1).

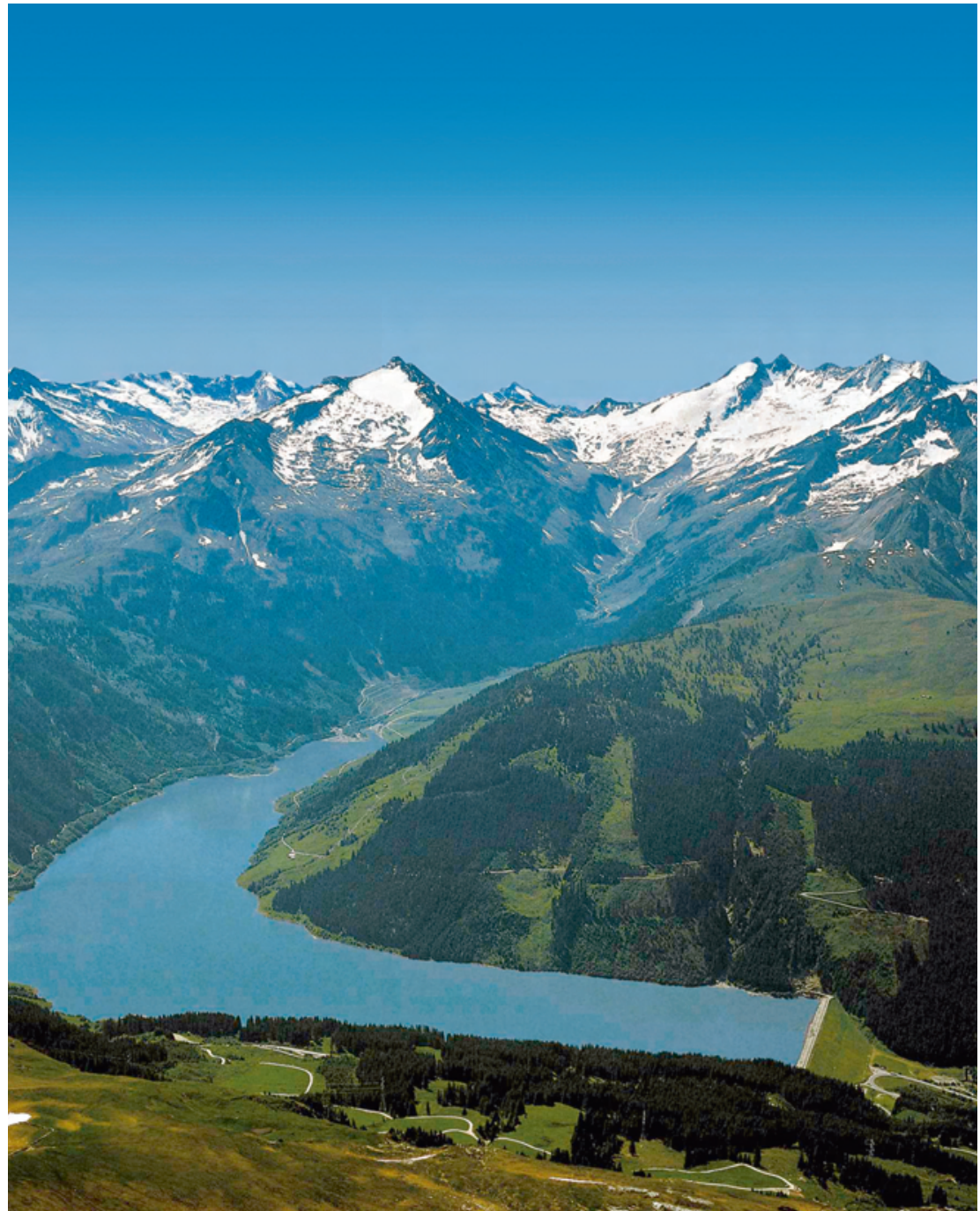

Abb. 1: Damm und Speicher Durlaßboden.

Technische Daten:

Höhe der Dammkrone: $\quad$ 1411,0 m ü.A.

Kronenlänge:

Kronenbreite:

Maximale Basisbreite:

Kubatur der Schüttung:

Die Gründung des Dammes erfolgte:

- an der orographisch linken Flanke auf gestörtem und aufgelockertem Grünschiefer

- in Talmitte auf einer $100 \mathrm{~m}$ starken Talauffüllung aus Kiessand und Schluff und

$5,5 \mathrm{~m}$

$340,0 \mathrm{~m}$

5 Mio. $\mathrm{m}^{3}$

- an der orographisch rechten Flanke in einer großräumigen Sackungsmasse aus Phyllit und Kalkphyllit

Eine Abdichtung bis zum Fels war in der Talmitte aus technisch-wirtschaftlichen Gründen nicht möglich, daher wurde der Dichtschirm in diesem Bereich in eine darüberliegende, weitgehend undurchlässige Schluffschicht eingebunden (Abb. 2)

Die Unterströmung des Dammes unterhalb des Schluffhorizontes wurde in das Entwurfskonzept miteinbezogen. Oberhalb dieser Schlufflage erfolgt der 


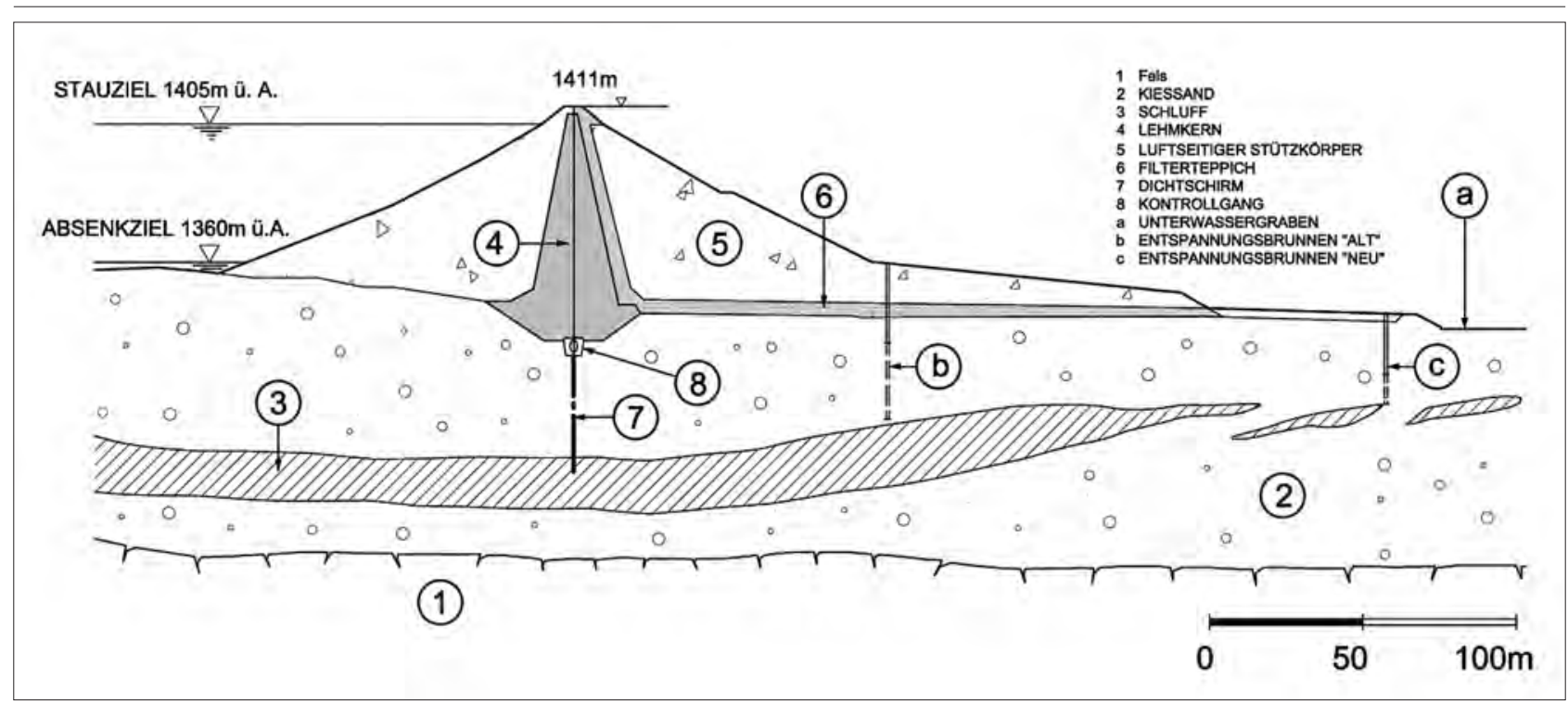

Abb. 2: Typischer Damm - Querschnitt.

Druckabbau über einen mehrreihigen Dichtschirm in der Dammachse und den Entspannungsbrunnen am Fuße des luftseitigen Stützkörpers. Im luftseitigen Dammvorland wurde zur Erhöhung der Auftriebssicherheit ein Flächenfilter mit einer Druckbank angeordnet.

Der in der Dammachse auf Niveau $1.300 \mathrm{~m}$ ü. A. festgestellte Schluffhorizont konnte bis etwa $330 \mathrm{~m}$ luftseitig der Dichtungsebene des Dammes auf etwa gleichbleibendem Niveau verfolgt werden, sodass sich projektsgemäß oberhalb und unterhalb der Schluffzone ein unterschiedliches Strömungspotential ergab.

\section{Druckverhältnisse $a b$} Inbetriebnahme im Jahr 1968 bis 1990

Im oberen Bereich wird das Strömungspotential des durch den Dichtungsschirm sickernden Wassers rasch abgebaut und beträgt etwa 50 m luftseitig des Dichtschirmes nur noch $22 \%$.

In den Entspannungsbrunnen am Dammfuß und im Flächenfilter wird das nunmehr mit einem kleinen Fließgefälle anströmende Wasser gesammelt, so dass die seichten Piezometer (PM 7, PM 9 und PM 10) luftseitig der Brunnen nur mehr wenig auf den Speicherspiegel reagieren.

Aufgrund der Abnahme der Gesamtsickerwassermenge von rd. 36l/s auf rd. 281/s in den Jahren 1985 bis 1989 bei den erwähnten Entspannungsbrunnen und einer leichten Absenkung des Grundwasserspiegels, kann in diesem Bereich auf eine Reduktion der Durchlässigkeit des Dichtschirmes durch Selbstdichtung geschlossen werden.
Der Dichtkern selbst kann als praktisch dicht angesehen werden (Summe Sickerwasser im Kontrollgang des Dammes $<2,5 \mathrm{l} / \mathrm{s}$ ).

In den tiefer liegenden Schichten nimmt das Strömungspotential im unbehandelten Kiessand deutlich langsamer ab. Die vorliegende Strömung wird von den über der Schluffschichte angeordneten Entwässerungseinrichtungen im Damm projektsgemäß wenig oder nicht erfasst. Dementsprechend sind im Unterwassergraben, etwa $330 \mathrm{~m}$ luftseitig der Dichtebene bzw. $230 \mathrm{~m}$ vom Dammfuß entfernt, immerhin noch $14 \%$ des Gesamtpotentials (Messung in rd. $20 \mathrm{~m}$ Tiefe) wirksam.

Unter Bezugnahme auf vorliegende Bohrkernauswertungen (Piezometerbohrungen im Jahre 1986) verliert sich dort der definierte Schluffhorizont, das Wasser strömt daher im gemischtkörnigen Kiessand von unterschiedlicher Durchlässigkeit nach oben und entspannt sich in Form von Quellen und Sandauftrieben an der Unterwassergrabensohle.

In diesen Bereichen ergaben sich bei Vollstau minimale Grundbruchsicherheiten von $s=1,6$ und für die Austrittsgradienten Werte bis zu 0,8.

Aufgrund dieser Erkenntnisse wurden im Jahr 1988 Überlegungen angestellt, mit entsprechenden Maßnahmen eine Ausspülung von Feinteilen aus dem Untergrund zu verhindern, die hydraulischen Grundbruchsicherheiten zu erhöhen und die Austrittskoeffizienten abzusenken.

Als Ergebnis wurde in Zusammenarbeit mit Herrn Prof. Breth (TU Darmstadt) ein Projekt, basierend auf der Errichtung zusätzlicher Entspannungsbrunnen im Bereich des Unterwassergrabens erstellt.

\section{Errichtung von zusätzlichen Entspannungsbrunnen}

\subsection{Grundsatzüberlegungen}

Nach umfassenden Untersuchungen und Überlegungen wurde im Einvernehmen mit Herrn Prof. Breth beschlossen, fünf zusätzliche Brunnen am Ufer des Unterwassergrabens $(300 \mathrm{~m}$ bis $350 \mathrm{~m}$ luftseitig der Dammachse) anzuordnen. Die Brunnen sollten das unter dem Talboden und in der rechten Talflanke abfließende Wasser aufnehmen, die zu diesem Zeitpunkt nach oben gerichtete Fließrichtung umlenken, Bodenausspülungen verhindern und eine verbesserte Erfassung und Überwachung des Grundwasserabflusses ermöglichen.

\subsection{Druckverhältnisse nach de Brunnenerrichtung}

Durch den Absenkeffekt der neuen Entspannungsbrunnen wurde das seinerzeitige Druckgefälle ermittelt aus den Piezometern PM K1/PM K2 (Brunnenprofil am Dammfuß) bis PM K17 (Unterwassergraben)

von $\frac{1373-1350}{230}=10 \%$ auf $\frac{1373-1345}{230}=12 \%$ vergrößert

Durch die Errichtung der neuen Brunnen trat eine Umverteilung der Sickerwässer 
auf - die bestehenden 7 Brunnen am Dammfuß zeigten eine Abnahme der Wasseraustritte, die sich zu den neuen Brunnen am Ufer des Unterwassergrabens hinverlagerte.

Die Filtergeschwindigkeit des Grundwassers erhöhte sich durch diese Maßnahmen

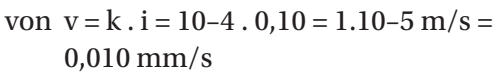

von $\mathrm{v}=\mathrm{k} \cdot \mathrm{i}=10-4 \cdot 0,10=1 \cdot 10-5 \mathrm{~m} / \mathrm{s}=$ $0,010 \mathrm{~mm} / \mathrm{s}$

auf $\mathrm{v}=10-4 \cdot 0,12=1,2 \cdot 10-5 \mathrm{~m} / \mathrm{s}=$ $0,012 \mathrm{~mm} / \mathrm{s}$

Die Gefahr von Erosionsvorgängen ist be den vorliegenden kleinen Fließgeschwindigkeiten dennoch auszuschließen. Ein internationaler Vergleich mit vergleichbaren Dämmen zeigt, dass Filtergeschwindigkeiten bis $\mathrm{zu} \mathrm{v}=3 \mathrm{~mm} / \mathrm{s}$ als unbedenklich gelten.

\subsection{Ausführungsprojekt}

Im Zeitraum von Mitte April bis Ende Mai 1990 wurden im Uferbereich des Unterwassergrabens die 5 Entspannungsbrunnen DREB 8, 9, 10, 11 und 13 errichtet.

Die Brunnenbohrungen wurden mit einem Durchmesser von $600 \mathrm{~mm}$ abgeteuft, die Brunnenrohre mit einer Nennweite von $250 \mathrm{~mm}$ festgelegt. Dabei wurden in den Filterstrecken Schlitzbrückenrohre (Typ: Hagusta) mit einem $3 \mathrm{~cm}$ starken Klebefiltermantel (Korngröße 2-3 mm) in Tiefen zwischen $20 \mathrm{~m}$ und $30 \mathrm{~m}$ unter der Geländeoberkante versetzt. Der verbleibende Restraum zwischen der Bohrlochwandung und dem Filterrohr wurde mit Filtersand (Ø 0,7-1,2 mm) verfüllt.
Zwischen der ca. 4,0 m tiefen Brunnenstube und den Filterrohren sind auf ca. 16,0 $\mathrm{m}$ Länge jeweils Vollrohre eingebaut, die als Abschluss mit einem Schieber und mit einer Druckmesseinrichtung versehen wurden. Von den Brunnenkammern wird das Wasser über Ableitungsrohre in den Unterwassergraben abgeleitet (Abb. 3).

Im Bereich des Unterwassergrabens zeigte sich nach der Errichtung der neuen Entspannungsbrunnen (1990) in der darauffolgenden Stauperiode eine Erhöhung der Auftriebssicherheit von $\mathrm{s}=1,60$ auf $\mathrm{s}=2,10$.

Die Austrittsgradienten verringerten sich vom Größtwert ia=0,80 auf ia=0,58. In der ersten Stauperiode wurde von den fünf neuen Entspannungsbrunnen in Summe 13l/s Wasser abgeführt (ca. $20 \%$ der theoretisch ermittelten Menge). Die Piezometer im luftseitigen Vorland zeigten in Abhängigkeit vom Abstand zu den Brunnen eine Druckhöhenreduktion zwischen $0,2 \mathrm{~m}$ und $5,0 \mathrm{~m}$.

In der folgenden Stauperiode (1991/92) wurde bei den fünf Entspannungsbrunnen ein Rückgang der Wasseraustritte um ca. $25 \%$ registriert - die Druckhöhen bei den Piezometern stiegen nahezu wieder auf das Niveau vor der Brunnenerrichtung.

Aufgrund dieser Entwicklung wurde während des ersten nachfolgenden Tiefstaues (Mai, Juni 1992) eine relativ aufwendige Schockentsandung der Brunnen vorgenommen. Mit dieser Maßnahme wurde eine stark verbesserte Wirkung der neuen Entspannungsbrunnen erreicht.

In der Stauperiode 1992/93 wurde im Vergleich zur vorangegangenen wiederum eine höhere Wasserförderung bei den

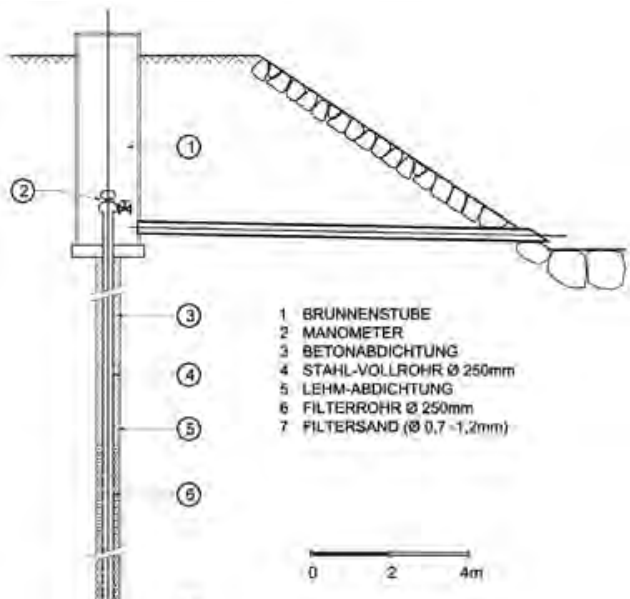

(7)

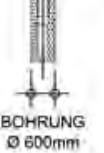

Abb. 3: Schnitt durch Entspannungsbrunnen.

neuen Entspannungsbrunnen und eine deutliche Abminderung der Druckhöhen im luftseitigen Vorland erreicht.

Dazu wurde in Jahr 1992 ein weiterer Brunnen in ähnlicher Bauweise etwa $350 \mathrm{~m}$ luftseitig der Dichtebene des Dammes ausgehend von der Sohle des Unterwassergrabens errichtet.

Da in dieser Stauperiode aus energiewirtschaftlichen Gründen eine max. Staukote von nur $1994 \mathrm{~m}$ ü. A. erreicht wurde, sind die nachfolgenden Vergleichswerte für einen Stau von ca. 1402 m ü. A. extrapoliert worden. Aus dieser Hochrechnung ergab sich für die neuen Entspannungsbrunnen eine Sickerwassermenge von ca. 291/s - und damit die dreifache

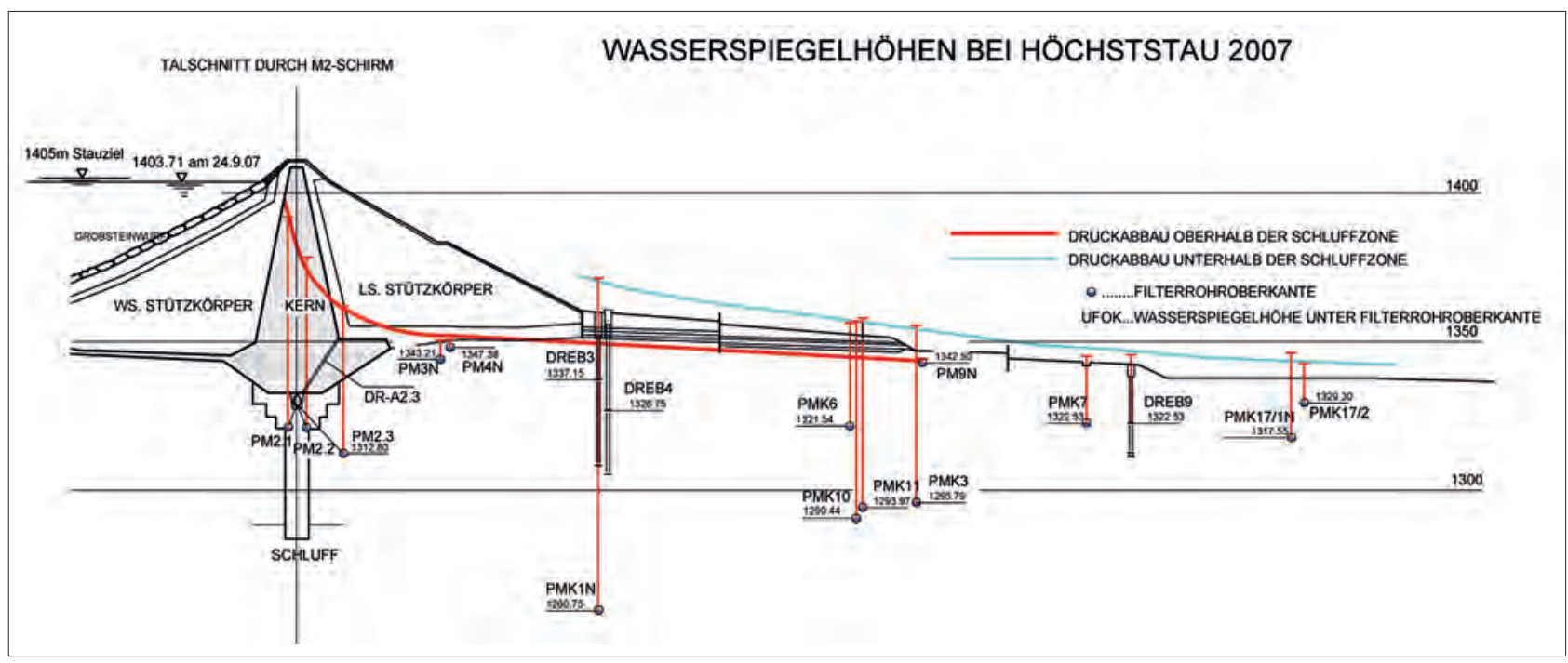

Abb. 4: Druckhöhen im Regelquerschnitt. 


\section{originalarbeit}

Menge gegenüber der ersten Stauperiode nach der Errichtung (1991).

Die Druckverhältnisse im luftseitigen Vorland zeigten bei besagter Hochrechnung (Extrapolation für Staukote $1402 \mathrm{~m}$ ü. A.) ebenfalls Druckreduktionen bis zu 8,0 m WS (Druckbankbereich: PM K 7). Im messtechnisch verfolgten Abschnitten des Unterwassergrabens ergaben sich bezogen auf das die vorangegangenen Messungen Druckabminderungen um nahezu 6,0 m WS (PM K 16).

Der zum jetzigen Zeitpunkt vorliegende Druckhöhenverlauf in einem Tallängenschnitt unterschieden nach der Auswertungen unter- und oberhalb der Schluffzone ist der Abbildung $4 \mathrm{zu}$ entnehmen.

Die in der Stauperiode 1992/93 über die Extrapolation ermittelten Werte wurden in den nachfolgenden Jahren bis zum heutigen Zeitpunkt aus den Messungen bei hohen Staukoten bestätigt. (Abb. 4). Mit den beschriebenen Maßnahmen konnten die Druckverhältnisse und damit die kritischen Auftriebssicherheiten und Austrittsgradienten entscheidend verbessert werden.

Die minimale Grundbruchsicherheit im Bereich des Unterwassergrabens beträgt nunmehr $\mathrm{s}=3,1$ und der höchste Austrittsgradient konnte auf ia $=0,36$ abgesenkt werden.

\section{Schlussbemerkungen:}

Die Errichtung der 6 Entspannungsbrunnen im Bereich des Unterwassergrabens erbrachte mit der Durchführung der nachträglichen Entsandung eine entscheidende Verbesserung der kritischen Druckverhältnisse im luftseitigen Vorland.

Bis zum heutigen Zeitpunkt zeigt sich nach den Maßnahmen 1990 bzw. 1992 ein nahezu unveränderter Zustand, d.h. die positiven Auswirkungen sind als langfristige Verbesserung zu betrachten.

Die Gesamtsickerwassermenge aus dem „alten“ und „neuen“ Entspannungsbrunnen blieb unverändert - durch die aufgetretene Umverteilung konnte jedoch ein äußerst positiver Effekt im weiteren luftseitigen Vorland erzielt werden.

Durch die umfassende Überwachung und laufende Beurteilung des Langzeitverhaltens des Erddammes Durlaßboden und des Umfeldes konnten als Reaktion auf Veränderungen zeitgerecht bauliche Maßnahmen vorgenommen werden und damit die Sicherheit der Gesamtanlage deutlich erhöht werden.

\section{Korrespondenz:}

Dipl. Ing. Alois Leobache

Verbund - Austrian Hydro Power AG

Am Hof $6 a$

1010 Wien

\section{LITERATUR}

Large Dams in Austria (1977)

Dams in Austria Vienna (1991) 